PROCEEDINGS OF THE

AMERICAN MATHEMATICAL SOCIETY

Volume 132, Number 10, Pages 3027-3034

S 0002-9939(04)07548-3

Article electronically published on May 12, 2004

\title{
COMMUTATIVE SUBALGEBRAS OF THE CORONA
}

\author{
DAN KUCEROVSKY
}

(Communicated by David R. Larson)

\begin{abstract}
We use methods of noncommutative functional analysis to extend the range of the usual functional calculus, for certain subalgebras of the corona. In particular, we construct corona projections with interesting properties.
\end{abstract}

\section{INTRODUCTION}

It may reasonably be said that the application of methods of algebraic topology to operator theory began with the work of Brown, Douglas, and Fillmore [1], followed by the fundamental papers of Kasparov [7, 8]. The very first steps in this direction, however, are probably due to Busby [2].

The first major success of these topological methods was the classification of the (approximately) abelian $C^{*}$-algebras generated by essentially normal operators. In this note, we apply ideas not far removed from those of Brown, Douglas, and Fillmore to study the nature of more general abelian subalgebras of the corona algebras of separable and stable $C^{*}$-algebras.

Recall that, in [5], a property of extensions called the purely large property was introduced and was proven to characterize absorbing extensions. The purely large condition is an algebraic condition on a subalgebra of a multiplier algebra associated with a given essential extension. An essential extension of $A$ by $B$ is said to be purely large if the extension algebra satisfies the purely large condition as a subalgebra of the multipliers of the canonical ideal, $B$. (The exact definition will be given later, as part of Theorem 1.1.) We recall that the introduction of the absorption property for extensions was originally motivated by the observation that extensions form a semigroup, under so-called Brown-Douglas-Fillmore addition. By definition, the BDF sum of (the Busby maps of) two essential extensions $\tau_{1}, \tau_{2}$ is

$$
a \mapsto w_{1} \tau_{1}(a) w_{1}^{*}+w_{2} \tau_{2}(a) w_{2}^{*}
$$

where $w_{1}, w_{2}$ are isometries generating a copy of $O_{2}$ in the multipliers. Such a copy of $\mathrm{O}_{2}$ will exist if the canonical ideal $B$ is stable, and happily, the Cuntz algebras $O_{n}$ have a universal property that ensures that a unital copy of $\mathrm{O}_{2}$ in the multipliers is unique up to conjugation by a multiplier unitary. Hence the above addition operation is unique up to unitary equivalence classes of extensions. Originally, a theorem

Received by the editors April 16, 2003.

2000 Mathematics Subject Classification. Primary 46L85; Secondary 47A60, 46L80.

Key words and phrases. KK-theory, absorbing extensions, functional calculus, corona $C^{*}-$ algebras.

This research was supported by NSERC, under grant \# 228065-00. 
due to Voiculescu [12] was used to show that in certain situations, extensions whose Busby maps lift to homomorphisms into the multipliers are actually algebraically trivial in the semigroup. Extensions that lift to homomorphisms in this sense are usually therefore called "trivial extensions." With this terminology, we can define the absorption property by saying that a semisplit extension $\tau$ is absorbing if the semigroup sum of $\tau$ with any trivial extension is unitarily equivalent to $\tau$, or, even more briefly, that an extension is absorbing if trivial extensions are algebraically trivial with respect to the given extension.

In a previous paper [5], by means of a generalization of the theorem due to Voiculescu (and further developed by others), we showed that the following conditions are equivalent.

Theorem 1.1. Let $A$ and $B$ be separable $C^{*}$-algebras, with $B$ stable and $A$ unital. Let $0 \longrightarrow B \longrightarrow C \longrightarrow A \longrightarrow 0$ be an essential unital extension with weakly nuclear completely positive splitting morphism $s: A \longrightarrow C$. Then the following are equivalent:

i) The extension absorbs trivial nuclear extensions.

ii) The extension algebra has the purely large property, i.e., the property that, for every positive element $c \in C^{+}$that is not zero in $C / B$, the hereditary subalgebra that it generates, $\overline{c B c}$, contains a stable subalgebra that is full in $B$.

We can say that an element of the corona, $x$, is purely large if $C^{*}(x)$ is in the image of an absorbing extension, or equivalently, if every lifting of $C^{*}(x)$ to the multipliers has the property described in the second clause of the above theorem.

Note that in the above theorem, it is not assumed that $B$ is simple. However, if $B$ is not simple, the algebra $C$ then has to be full with respect to $B$, so to speak, in a very strong sense. We next proceed to construct such an algebra.

First, fix a copy of $\mathrm{O}_{2}$ in the multipliers, and let us agree that (Brown-DouglasFillmore) addition of extensions will be with respect to this copy. Words in the generators $w_{1}$ and $w_{2}$ of $O_{2}$ can be used to construct an infinite sequence of isometries, $v_{1}, v_{2}, v_{3}, \cdots$ that generate a copy of $O_{\infty}$. Let $B$ be of the form $B_{0} \otimes \mathcal{K}$, where $B_{0}$ is unital, and define a map $\bar{\delta}: \ell^{\infty} \longrightarrow \mathcal{M}(B)$ by $\left(\lambda_{i}\right) \mapsto \sum_{1}^{\infty} \lambda_{i} e_{i i}$, with convergence understood to be in the strict topology. Clearly this is a homomorphism, and composing with the natural quotient map $\pi: \mathcal{M}(B) \longrightarrow \mathcal{M}(B) / B$, we find that the kernel of $\pi \circ \bar{\delta}$ is exactly the space of sequences converging to zero. Thus we have an injective homomorphism, denoted by $\delta$, from $\ell^{\infty} / c_{0}$ to $\mathcal{M}(B) / B$, the image of which we denote by $\bar{W}$.

Noticing that $c_{0}$ can be thought of as $C_{0}(\mathbb{N})$, where the integers $\mathbb{N}$ have the usual discrete topology, we see that the space of characters of $\left.\ell^{\infty} / c_{0} \cong \mathcal{M}\left(C_{0}(\mathbb{N})\right) / C_{0}(\mathbb{N})\right)$ is $\beta(\mathbb{N}) \backslash \mathbb{N}$, where $\beta(\mathbb{N})$ is the Stone-Čech compactification of the natural numbers. Hence, by the Gelfand theorem, $\delta$ can be identified with a map from $C(\beta(\mathbb{N}) \backslash \mathbb{N}$ into $\mathcal{M}(B) / B$. As a map into the multipliers, it maps $C(\beta(\mathbb{N}))$ into $\mathcal{M}(B)$.

Let us denote the image of $\delta$ in $\mathcal{M}(B) / B$ by $W$.

Proposition 1.2. Let $B$ be a stable and $\sigma$-unital $C^{*}$-algebra. The image $\bar{W}$ of the map $\delta: C(\beta(\mathbb{N})) \longrightarrow \mathcal{M}(B)$ is purely large with respect to $B$.

Proof. Choose some positive element $\left(\lambda_{i}\right)$ of $\ell^{\infty}$ that is not in $c_{0}$. We must show that the hereditary subalgebra $\overline{\delta\left(\left(\lambda_{i}\right)\right) B \delta\left(\left(\lambda_{i}\right)\right)}$ is stable and full in $\overline{B c B}$. 
Let $\left(e_{i i}\right)$ be the projections defining the map $\delta$. The multiplier element

$$
c:=\sum \lambda_{j} e_{i i}
$$

is also full in $B$, and it is not difficult to show that the hereditary subalgebra $H:=\overline{c B c}$ is therefore full in $B$.

We shall show that the multipliers of $H$ contain an infinite family of equivalent, orthogonal projections summing to the identity (in the strict topology), which will then be used to show that $H$ is stable.

Let $L$ be the support projection of $c$, which is the multiplier projection obtained by applying $\delta$ to the sequence

$$
s_{j}:=\left\{\begin{array}{lll}
0 & \text { if } & \lambda_{j}=0 \\
1 & \text { if } \quad \lambda_{j} \neq 0 .
\end{array}\right.
$$

Since $\lambda_{j}$ does not go to zero as $j \longrightarrow \infty$, this sequence $\left(s_{j}\right)$ is nonzero for infinitely many $j$. It is clear that $H=L B L$, and hence the multipliers $\mathcal{M}(H)$ of $H$ are equal to the multiplier corner $L \mathcal{M}(B) L$. Let us now replace the $\left(e_{i i}\right)$ by the infinite subsequence, still denoted $\left(e_{i i}\right)$, corresponding to the nonzero $\lambda_{i}$. Since the projections $e_{i i}$ are majorized by $L$, we see that the $e_{i i}$ are projections in $L \mathcal{M}(B) L$.

Thus, the $e_{i i}$ are in the multipliers of $H$, are equivalent, and sum to 1 strictly. It follows that $H$ is stable.

Proposition 1.3. Given a unital abelian separable subalgebra $A$ of $\mathcal{M}(B) / B$, there is a commutative diagram

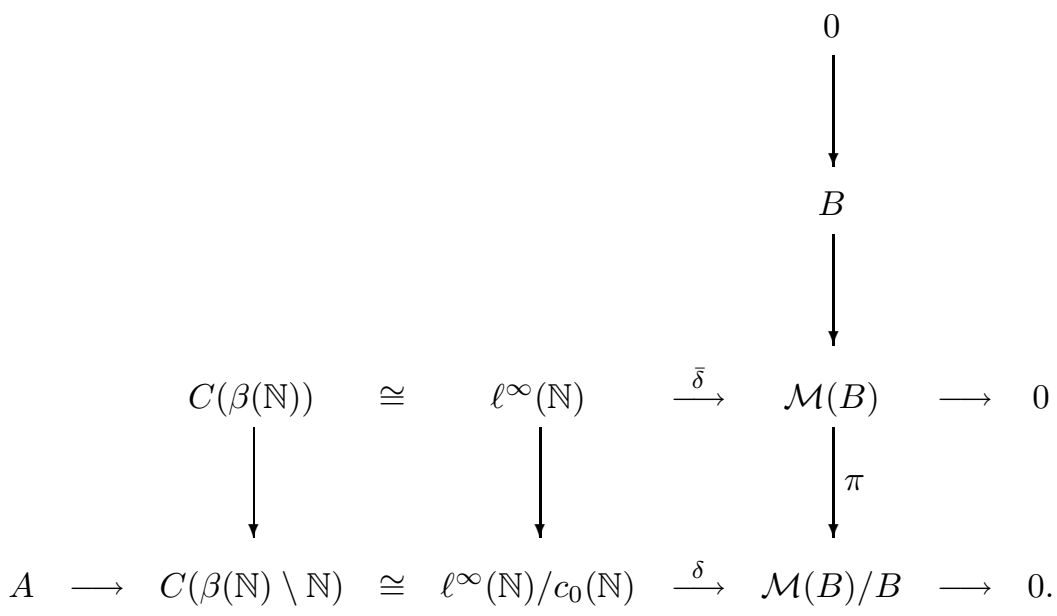

The (Busby) map from $A$ to $\mathcal{M}(B) / B$ defined by this diagram is injective, purely large, and nuclear. It is trivial if and only if $A$ is separable.

Proof. By the Gelfand theorem, $A=C(X)$ for some compact Hausdorff space $X$ with a countable dense subset. This countable dense subset gives us a continuous mapping $h: \mathbb{N} \longrightarrow X$ with dense image in $X$. Using the fundamental property of Stone-Cech compactifications to extend $h$ uniquely to a map $\beta(h): \beta(\mathbb{N}) \longrightarrow X$, we see that the image of $\beta(h)$ is compact, hence closed, and dense in $X$. Therefore the image is all of $X$, and the pullback of $\beta(h)$ is thus an injective homomorphism of $A$ into $C(\beta(\mathbb{N})$ ), which we then compose with $\bar{\delta}$ to obtain (the lifting of) a trivial Busby map. 
Recall that the extension algebra $C$ associated with the constructed Busby map, $\tau$, is by definition the pullback

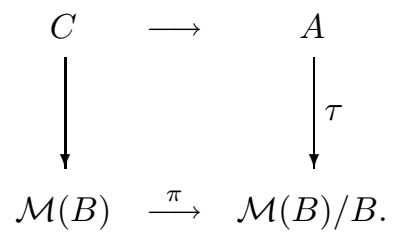

Since $\tau$ has no kernel, we may as well regard $C$ as being identified with its image in $\mathcal{M}(B)$, and then by comparing with the above diagram, we see that the extension algebra is in fact a subalgebra of the image $W$ of $\bar{\delta}$ in $\mathcal{M}(B)$. The image algebra $W$ is purely large by Lemma 1.2 and the purely large property passes to subalgebras, so that the extension is purely large as claimed.

In order to avoid making our statements too technical, we have generally tried to avoid the language of $K$-theory. For the purposes of developing a functional calculus, we are primarily interested in the case of singly-generated abelian subalgebras, and the following lemma shows that most such elements are $K$-theoretically trivial. Hence only the purely large condition will need to be verified.

Lemma 1.4. If $A$ is a separable abelian $C^{*}$-algebra generated by a single selfadjoint element a with connected spectrum $[0,\|a\|]$, then copies of $A$ in a corona $\mathcal{M}(B) / B$ lift to the multipliers by a homomorphism.

Proof. Taking the image of the generator of $A$ in the corona, we can lift it to a selfadjoint element in the multipliers. Doing this might in general increase the spectrum, but we can apply a suitable continuous function to make sure that the lifting has the same spectrum as the original element. Then we can use the Gelfand theorem to contruct a homomorphism $s: A \longrightarrow \mathcal{M}(B) / B$ such that $\pi \circ A=\operatorname{Id}_{A}$.

Assembling the above lemmas, we see that if $x$ is a selfadjoint element of the corona that has continuous spectrum, and the purely large property, then the trivial extension $\iota: C^{*}(x) \longrightarrow \mathcal{M}(B) / B$ and the extension $\kappa: C^{*}(x) \longrightarrow W \subset \mathcal{M}(B) / B$ from Proposition 1.3 are both trivial absorbing extensions. They are thus in the same Kasparov theory equivalence class, and being absorbing, they are equivalent up to unitary equivalence by a multiplier unitary. Therefore there is a multiplier unitary $U_{x}$ such that $U_{x} a U_{x}^{*}=\kappa(a)$, meaning that $C^{*}(x)$ can be moved into $W$ by conjugation by $U_{x}$.

Definition 1.5. The image of $W$ in $C^{*}(x)^{\prime}$ under the map $c \mapsto U_{x}^{*} c U_{x}$ is called the algebra associated with $C^{*}(x)$.

Next on the agenda is to consider the implications of this above observation for the functional calculus of $x$. Loosely speaking, we may hope that the associated algebra of $x$ is large enough to allow a few constructions that at first glance would seem to require passing to the second dual. We have seen that we can transport $x$ into $W$ by a unitary equivalence, and since the image of $x$ is a function on a totally disconnected compact Hausdorff space, we may be able to construct elements of $W$ that have interesting properties with respect to the image of $x$. If those properties can be expressed in terms of equations or commutation relations, we can then transport back (conjugating by $U_{x}^{*}$ ) to obtain elements that commute with the originally given $x$, and that still have the properties of interest with respect to the 
original $x$. Of course, the newly constructed elements of what we call the associated algebra belong to $C^{*}(x)^{\prime} \cap \mathcal{M}(B) / B$, and may or may not be in $C^{*}(x)^{\prime \prime}$. First we address the question of whether or not we obtain anything that cannot be done with the ordinary functional calculus. The next several results are largely a (not entirely trivial) restatement of topological properties of $\beta(\mathbb{N})$ in operator-theoretical terms.

Theorem 1.6. If $A_{1} \subset \mathcal{M}(B) / B$ is a purely large abelian subalgebra of $\mathcal{M}(B) / B$, and the spectrum of $f \in A_{1}$ is $[0,1]$, then $A_{1}$ can be enlarged to a separable, purely large abelian subalgebra $A_{2} \subset \mathcal{M}(B) / B$, containing a projection $p$ such that

i) any element of $A_{1}$ that acts as the unit on $f$ acts as the unit on $p$, and

ii) $p$ is orthogonal to any element of $A_{1}$ that is orthogonal to $f$.

Proof. Let $F_{1}=f^{-1}(1)$ and $F_{0}=f^{-1}(0)$. The sets $F_{1}$ and $F_{0}$ are closed, disjoint, nonempty subsets of the spectrum $[0,1]$ of $f$. Recalling (Proposition 1.3 ) that $\beta(\mathbb{N}) \backslash$ $\mathbb{N}$ maps continuously onto a compact set, we denote the inverse image of these two sets, in $\beta(\mathbb{N})$, by $K_{1}$ and $K_{0}$. By [13, 2.13], these two disjoint closed subspaces are separated by a partition of $\beta(\mathbb{N}) \backslash \mathbb{N}$ by two closed and open sets, $P_{1}$ and $P_{0}$. Defining a continuous function $p \in W$ by

$$
p(x):=\left\{\begin{array}{l}
0 \text { if } x \in P_{0}, \\
1 \text { if } x \in P_{1},
\end{array}\right.
$$

we see that if $f_{W}$ denotes the image of $f$ in $W$, and $g_{W}$ the image of a $g$ orthogonal to $f$, then the support set $\overline{\operatorname{Supp} g_{W}}$ is contained in $K_{0}$, and $p g_{W}=0$. The case of elements acting as the unit on $f$ is similar. We have yet to show that the algebra generated by $A_{1}$ and the image $U^{*} p U$ of $p$ is separable and purely large, but this follows from the next lemma.

Lemma 1.7. If $A_{1}$ is a purely large, separable abelian algebra, and $U$ is a multiplier unitary such that $U A_{1} U^{*} \subseteq W$, then the algebra generated by $A_{1}$ and $U^{*} w U$, where $w$ is in $W$, is still separable, abelian, and purely large.

Proof. First, note that the algebra generated by $A_{1}$ and $U^{*} w U$ is a subalgebra of $U^{*} W U$. However, $W$ is a purely large subalgebra of the corona, and the purely large property is clearly preserved by conjugation by multiplier unitaries, since $\overline{c B c}$ contains a stable and full subalgebra if and only if $\overline{U^{*} c U B U^{*} c U}$ does. The remaining claims are easily checked.

Proposition 1.8. Let $A_{1}$ be purely large, abelian, and separable. Let $f_{1}, f_{2}$ be two orthogonal elements of $A_{1}$ with spectrum $[0,1]$. We can enlarge $A_{1}$ to a separable, purely large, and abelian subalgebra of $\mathcal{M}(B) / B$ such that $f_{1}$ and $f_{2}$ are separated by a projection in the enlarged algebra.

Proof. Since $f_{1}$ and $f_{2}$ are orthogonal, the $C^{*}$-algebra they generate is really the same as the algebra generated by $f_{1} \oplus f_{2}$. By Lemma 1.4, this algebra is the image of a trivial absorbing extension, and we can inject $f_{1}$ and $f_{2}$ into $W$, by conjugation by a multiplier unitary. We still denote their images by $f_{1}$ and $f_{2}$. Since they are clearly orthogonal in $W$, the open sets $C_{i}:=\left\{x \mid f_{i}(x) \neq 0\right\}$ are disjoint. By [13. 3.24], sets of this type are separated by a partition of $\beta(\mathbb{N}) \backslash \mathbb{N}$ by two closed and open sets $K_{1}$ and $K_{2}$. Defining a continuous function $p \in W$ by

$$
p(x):=\left\{\begin{array}{l}
0 \text { if } x \in K_{2}, \\
1 \text { if } x \in K_{1},
\end{array}\right.
$$


we now transport $p$ back, obtaining a projection $p \in \mathcal{M}(B) / B$ in the associated algebra such that $p$ and $f_{i}$ commute. Since the algebra generated by $A$ and $p$ is separable, abelian, and is in the image of a purely large subalgebra by a multiplier unitary, we are done.

In the next proposition, $A$ can for example be any separable algebra generated by one or more orthogonal positive elements with the purely large property. We first need the following topological result.

Theorem 1.9 (Parovičenko [10, 14]). A totally disconnected compact F-space without isolated points, having weight $\aleph_{1}$, and such that every zero-set is a regular closed set is homeomorphic to $\beta(\mathbb{N}) / \beta$.

Proposition 1.10. Let $A$ be the image in the corona of a separable, trivial, purely large extension. Let $f$ be a positive element of $A$ with uncountable spectrum. Then:

i) There is a copy of $W$ in $A^{\prime}$ such that no nonzero element of this copy of $W$ annihilates $f$.

ii) There are uncountably many orthogonal projections in $W$.

iii) The hereditary subalgebra generated by $f$ cannot be faithfully represented on any separable Hilbert space.

Proof. If $\operatorname{sp} f$ is an uncountable subset of $[0,\|f\|]$, it is (being closed) the union of a perfect set and an at most countable set. Let $a, b>0$ be such that $[a, b]$ has nonzero intersection with this perfect set. Then, $f^{-1}[a, b]$ is an infinite closed subset of $X=\operatorname{sp} A_{1}$. Since $\beta(\mathbb{N})$ maps onto $X$, the inverse image of this closed subset is a closed subset $K$ of $\beta(\mathbb{N}) \backslash \mathbb{N}$. Since the closed and open sets form a basis for $\beta(\mathbb{N}) \backslash \mathbb{N}$, there is a closed and open set $K_{1}$ contained in $K$. This closed and open set $K_{1}$ cannot be finite, since a finite open set in a Hausdorff space contains an isolated point of the space, and $\beta(\mathbb{N}) \backslash \mathbb{N}$ has no isolated points. Since $K_{1}$ is closed and open in $\beta(\mathbb{N}) \backslash \mathbb{N}$, the continuous functions $C\left(K_{1}\right)$ can be extended to $C(\beta(\mathbb{N}) \backslash \mathbb{N})$ just by defining them to be zero outside $K_{1}$, and hence $C\left(K_{1}\right)$ is embedded in $W$ (by an injection). The image of $C\left(K_{1}\right)$ in $A^{\prime}$ under the unitary equivalence will, in fact, provide the claimed copy of $W$ in $A^{\prime}$. To see this, notice that an infinite closed and open subset of $\beta(\mathbb{N}) \backslash \mathbb{N}$ satisfies the hypotheses of Parovičenko's characterization of $\beta(\mathbb{N}) \backslash \mathbb{N}$, and hence is homeomorphic to $\beta(\mathbb{N}) \backslash \mathbb{N}$. It therefore follows that $C\left(K_{1}\right)$ is isomorphic to $W$. Since $K_{1}$ is in the support of the image of $f$ in the original algebra $W$, it is clear that no nonzero element of $C\left(K_{1}\right)$ can annihilate $f$. This proves the first claim.

To prove the second claim, notice that the countable infinite product $\times_{1}^{\infty} D$, where $D$ is a finite discrete space such as $\{0,1\}$, is compact (by Tychonoff), Hausdorff, and contains an uncountable set $\mathcal{B}$ of orthogonal elements. Moreover, it has a basis of cardinality $\aleph_{1}$. Hence, by the surjectivity statement in Parovičenko's theorem, there is an injection from $C(D)$ into $W$, and the characteristic functions of the elements of $\mathcal{B}$ give an uncountable orthogonal subset of $W$. This establishes the second part.

For the last part, notice that the orthogonal set of projections found in the previous paragraph will, since they are not annihilated by $f$, give an uncountable set of orthogonal elements of the hereditary subalgebra $\overline{f\left(\frac{\mathcal{M}(B)}{B}\right) f}$ generated by $f$ in the corona. This cannot happen if the hereditary subalgebra can be faithfully represented on a separable Hilbert space. 
Remark 1.11. Notice that we have also shown that no copy of $W$ can be faithfully represented on a separable Hilbert space. Since, by Proposition 1.2, a copy of $W$ exists in the corona of any stable separable $C^{*}$-algebra $\tilde{B}_{0} \otimes \mathcal{K}$, we have shown that no such corona algebra can be represented on a separable Hilbert space. (This was pointed out to us by Akemann. In fact, Akemann's example works even without stability, but on the other hand, our result gives much more precise information.)

One may optimistically ask if one could use Proposition 1.8 to construct a Borel function calculus based on projections, similar to that found in von Neumann algebras. The closed and open subsets do form a Boolean algebra, but it can be shown not to be complete. The smallest complete Boolean algebra is the one generated by the (co)-zero sets in $\beta(\mathbb{N}) \backslash \mathbb{N}$. In fact, it is of interest that a strictly increasing sequence of projections in $W$ can never have a least upper bound. We thus have the following interesting result (a reformulation of a result originally due to W. Rudin), reminiscent of Pedersen's CRISP property 11. One of the differences is that here, part of the conclusion is that the element constructed is neither less than nor greater than elements of some specified countable sequence $\left(C_{i}\right)$.

Proposition 1.12. [13, 2.24] The algebra of projections in $W$ has the property that, given countable (possibly finite) sequences $\left(A_{i}\right),\left(B_{i}\right)$, and $\left(C_{i}\right)$ such that

i) $A_{1}<\cdots<A_{k}<\cdots<B_{\ell}<\cdots<B_{1}$,

ii) $C_{n} \not \leq A_{\ell}$,

iii) $B_{\ell} \not \leq C_{n}$,

then there exists $D$ in $W$ such that

i) $A_{\ell}<D<B_{m}$,

ii) $D$ is not comparable to any $C_{n}$.

In particular, in between any two comparable but not equal projections $A$ and $B$, one can find another projection, which can moreover be taken incomparable to some projection that is not comparable with either $A$ or $B$.

Theorem 1.13. The functions with finite range are dense in $W$.

Proof. It is sufficient to consider only real-valued functions. The corona $\beta(\mathbb{N}) \backslash \mathbb{N}$ has topological dimension zero, and hence $W$ has Katětov dimension zero. Thus, if $R \subset C(\beta(\mathbb{N}) \backslash \mathbb{N})$ is a subring of real functions containing the constant functions, and having the property that $f^{2} \in R$ implies $f \in R$, then $R$ is dense in the selfadjoint part of $C(\beta(\mathbb{N}) \backslash \mathbb{N})$. In particular, the subring of real functions with finite range is such a ring, and hence is dense in the selfadjoint part of $W$.

The following corollary is a reasonably well-known general property of function algebras over totally disconnected Hausdorff spaces; see, for example, Goodner [6].

Corollary 1.14. The algebra $W$ is generated by its projections.

Theorem 1.15. The function algebra $W$ is algebraically closed: a polynomial equation with coefficients in $W$ has roots in $W$.

Proof. It is known [3] that a nontrivial polynomial $P(x, \lambda)=\lambda^{n}+a_{n-1}(x) \lambda^{n-1}+$ $\cdots+a_{0}(x)$ with coefficients $a_{i}(x)$ coming from a function algebra on a compact, totally disconnected Hausdorff space has at least one root $\lambda=f(x)$ in that function algebra. Since there is a unique factorization theorem and a division algorithm for polynomials over a commutative ring, $P(x, \lambda)=Q(x, \lambda) \cdot(\lambda-f(x))$. Since $Q(x, \lambda)$ will also have a root in $W$, we proceed by induction on the degree of $P$. 
Next, observing that since the canonical ideal $B$ is stable, the corona contains a lot of equivalent projections, and hence is stable under tensor products with finite matrix algebras, we have $M_{n}(\mathcal{M}(B) / B)=\mathcal{M}\left(M_{n}(B)\right) / M_{n}(B) \cong \mathcal{M}(B) / B$. Therefore, in $\mathcal{M}(B) / B$ there will be a lot of subalgebras of the form $C(X) \otimes M_{n}$. If the subalgebra $C(X)$ is in the image of an absorbing extension, as will always be the case if, for example, $B$ is purely infinite and simple, it seems interesting to consider the behaviour of the enveloping subalgebra, $W \otimes M_{n}$. We then have the following proposition, obtained by applying the results of [4] to our situation.

Proposition 1.16. Each invertible element of $M_{n} \otimes W$ has a logarithm in $M_{n} \otimes W$.

Corollary 1.17. The $K$-theory of $W$ is trivial in odd dimension: $K^{1}(W)=\{e\}$.

The other $K$-group is of course nontrivial, and is too large to be useful, being uncountably generated.

We thank Chuck Akemann for showing us an interesting example.

\section{REFERENCES}

1. L. Brown, R. Douglas, P. Fillmore, Unitary equivalence modulo the compact operators and extensions of $C^{*}$-algebras, Lecture Notes in Math. 345 (1973), 58-128. MR 52:1378

2. R.C. Busby, Double centralizers and extensions of $C^{*}$-algebras, Trans. Amer. Math. Soc. 132 (1968), 79-99. MR 37:770

3. D. Decard, C. Pearcy, On Algebraic Closure in Function Algebras, Proc. Amer. Math. Soc. 15 (1964), 259-263. MR 28:4379

4. D. Decard, C. Pearcy, On Continuous Matrix-Valued Functions on a Stonian Space, Pacific J. Math. 14 (1964), 857-869. MR 30:2356

5. G.A. Elliott, D. Kucerovsky, An abstract Voiculescu-Brown-Douglas-Fillmore absorption theorem, Pacific J. of Math. 198 (2001), 385-409. MR 2002i:46052

6. D.B. Goodner, The closed convex hull of certain extreme points, Proc. Amer. Math. Soc. 15 (1964), 256-258. MR 29:455

7. G.G. Kasparov, Hilbert $C^{*}$-modules: theorems of Stinespring and Voiculescu, J. Operator Theory 4 (1980), 133-150. MR 82b:46074

8. G.G. Kasparov, The operator $K$-functor and extensions of $C^{*}$-algebras, Math. USSR Izvestiya 16 (1981), 513-572. MR 81m:58075

9. J. Hjelmborg and M. Rørdam, On Stability of $C^{*}$-algebras, J. Funct. Anal. 155 (1998), 153170. MR 99g:46079

10. I.I. Parovičenko, On a universal bicompactum of weight $\aleph$, Soviet Mathematics, 4 (1963), 592-595. MR 27:719

11. G.K. Pedersen, The Corona construction, Proceedings 1988 GPOTS-Wabash conference, 1990, 49-92. MR 92e:46119

12. D.V. Voiculescu, A non-commutative Weyl-von Neumann theorem Rev. Roum. Pures Appl. 21 (1976), 97-113. MR 54:3427]

13. R.C. Walker, The Stone-Čech compactification, Springer, 1974. MR 52:1595

14. R.G. Woods, Co-absolutes of remainders of Stone-Čech compactifications, Pacific J. Math. 37 (1971), 545-560. MR 46:6300a

Department of Mathematics and Statistics, University of New BrunswickFredericton, Fredericton, New Brunswick, Canada E3B 5A3

E-mail address: dkucerov@unb.ca 